\title{
Reduced Diaphragm Excursion During Reflexive Citric Acid Cough Test in Subjects With Subacute Stroke
}

\author{
Yong-Min Choi MD, Geun-Young Park MD PhD, Yeonji Yoo MD, Donggyun Sohn MD, \\ YongJun Jang MD, and Sun Im MD PhD
}

\begin{abstract}
BACKGROUND: Diaphragm excursion is limited during respiratory maneuvers after a stroke. How the diaphragm is limited during reflexive coughs and affects the effectiveness of cough in stroke patients is unclear. This study aimed to measure reflexive cough strength by cough peak flow (CPF) induced by citric acid nebulization $(2.8 \mathrm{~mol} / \mathrm{L})$, record diaphragm excursions during reflexive coughs in stroke subjects at risk of silent aspiration, and compare these values with those of stroke subjects without risk of aspiration or dysphagia. METHODS: Twenty-one subjects with subacute stroke (mean stroke onset, 13.6 d) at risk of silent aspiration (penetration-aspiration scale, 8) and 21 stroke subjects without dysphagia or aspiration (controls) were included. Diaphragmatic excursions were assessed using real-time sonography in all subjects; the main outcome measure was reflexive CPF induced by citric acid nebulization. RESULTS: The median (interquartile range) values of citric acid-induced CPF values were significantly more reduced in the 21 subjects with silent aspiration $(45[0-83] \mathrm{L} / \mathrm{min})$ than in the control subjects $(97[66-162] \mathrm{L} / \mathrm{min})(P=.004)$. Diaphragmatic excursions during the reflexive coughs were also significantly reduced $(P<.001)$, although both groups had a similar range in the initial National Institutes of Health Stroke Scale scores and level of disability, as measured by the modified Barthel index. Citric acid-induced CPF was significantly correlated with the number of generated coughs $\left(r_{s}=0.69\right)$, voluntary cough CPF $\left(r_{s}=0.85\right)$, and degree of diaphragm excursion on both sides $\left(r_{s}=0.50\right.$ [hemiplegic] and $r_{s}=0.55$ [nonhemiplegic]) but not correlated with the degree of hemiparesis, National Institutes of Health Stroke Scale score, or modified Barthel index scores. The 6-month follow up revealed that 7 subjects in group A experienced aspiration pneumonia. CONCLUSIONS: Stroke subjects at risk of silent aspiration showed reduced $\mathrm{CPF}$ and more limited diaphragm excursion during the citric acidinduced reflexive cough test. (ClinicalTrials.gov registration NCT02080988.) Key words: aspiration pneumonia; cough; hemiplegia; stroke; ultrasonography; diaphragm excursion; reflexive cough; silent aspiration. [Respir Care 2017;62(12):1571-1581. (C) 2017 Daedalus Enterprises]
\end{abstract}

\section{Introduction}

A proper cough is particularly important for patients with swallowing difficulties. ${ }^{1,2}$ An ineffective cough mech-

Dr Choi is affiliated with the Department of Rehabilitation Medicine, Keimyung University, Donsang Medical Center, Deaegu, Korea. Drs Park, Yoo, Sohn, Jang, and Im are affiliated with the Department of Rehabilitation Medicine, Bucheon St. Mary's Hospital, College of Medicine, Catholic University of Korea, Seoul, Korea.

This work was supported by the Institute of Clinical Medicine Research of Bucheon St. Mary's Hospital (Seoul, Korea) Research Fund (grant BCMC13AH06). The statistical consultation was supported by a grant from the Korea Health Technology R\&D Project through the Korea anism may predispose these patients to respiratory complications. ${ }^{3}$ Voluntary cough and reflexive cough are pertinent in airway protection and in preventing aspiration pneumonia. ${ }^{4}$

\footnotetext{
Health Industry Development Institute, which is funded by the Ministry of Health and Welfare, Republic of Korea (Seoul, Korea; grant HI14C1062). The authors have disclosed no conflicts of interest.

Correspondence: Sun Im MD PhD, Department of Rehabilitation Medicine, Bucheon Street Mary's Hospital, College of Medicine, Catholic University of Korea, 327 Sosa-ro, Bucheon-si, Gyeonggi-do 14647, Korea. E-mail: lafoliamd@gmail.com or lafolia@catholic.ac.kr.
}

DOI: $10.4187 /$ respcare. 05488 


\section{Diaphragm Excursion During Reflexive Cough in Subjects With Stroke}

Voluntary cough reflects a person's capacity to voluntarily move aspirated particles from the lower airway to above the vocal cords through a cough maneuver. ${ }^{5}$ Stroke weakens voluntary cough and thereby increases the risk of aspiration. ${ }^{6}$ The close relationship between swallowing and protective cough is crucial, but the mechanism of these interconnected systems needs to be elucidated. A previous study $^{7}$ suggests that stroke subjects with dysphagia and aspiration may have voluntary cough strength and have limited diaphragm excursion needed to generate sufficient positive pressure to produce a strong cough.

Involuntary cough (ie, reflexive cough) is produced reflexively and protects against tracheobronchial aspiration. ${ }^{8}$ Reflexive cough is important in ensuring adequate airway protection and clearing secretions or foreign food material that may enter the airway, and it is essential for airway protection during swallowing. ${ }^{9}$ The integrity of reflexive cough can be tested using tussive agents, such as citric acid or tartaric acid. ${ }^{10-13}$ After a stroke event, cough sensory sensitivity to produce a reflexive cough becomes impaired. ${ }^{14}$ The citric acid cough test is useful in screening patients at risk of silent aspiration.,5,11 Various studies have assessed diaphragm excursion in hemiplegic stroke $7,15,16$; however, how diaphragm excursion is affected during reflexive cough in stroke patients is unknown. Reflexive cough is crucial in airway protection; therefore, proper understanding of how the diaphragm is affected in individuals with stroke is crucial.

Adequate afferent sensory inputs from the laryngopharyngeal area, which are crucial to initiate swallowing, also protect the airway by triggering reflexive cough whenever food or saliva enters the airway. Silent aspiration is a consequence of impairment in the pharyngeal afferents. Silent tracheobronchial aspiration can lead to a 13-fold increased risk of developing pneumonia. ${ }^{17}$ What has received less attention is the role of these afferents in regulating the strength of inspiratory and expiratory efforts during reflexive coughing. ${ }^{18,19}$ A previous study ${ }^{10}$ has shown that expiratory muscle activation during reflexive cough is reduced in people with decreased pharyngeal sensation after laryngectomy. However, whether the same principle can be applied to the diaphragm, a main inspiratory muscle with an important role in cough generation, has not been established.

Proper understanding of how the reflexive cough output is affected after stroke is crucial for implementing proper rehabilitative treatment strategies and preventing respiratory complications. The present study thus aimed to (1) test cough strength during reflexive cough, as assessed with a peak flow meter using a fixed dosage of citric acid;

(2) record diaphragm excursion during these coughs; and (3) determine how these are affected in stroke patients at risk of silent aspiration.

\section{QUICK LOOK}

\section{Current knowledge}

Stroke patients at risk of aspiration have reduced cough strength and limited diaphragm excursion during voluntary coughing. These patients have diminished cough sensitivity during reflexive cough tests using tussive agents; however, it is unclear how weak these coughs are and how diaphragm excursion is affected during reflexive coughing. Because a reflexive cough is related to a cough during swallowing, cough peak flow (CPF) could be clinically relevant.

\section{What this paper contributes to our knowledge}

Stroke subjects with a high risk of aspiration had diminished diaphragm excursions with reduced CPF during the citric acid reflexive cough tests compared with those with no risk of aspiration. Even in the presence of positive cough signs during the reflexive cough test, those at risk of silent aspiration still had reduced CPF. Our results highlight the need to measure cough strength in an objective manner in addition to the impaired cough sensitivity during standard reflexive cough tests in stroke patients at risk of silent aspiration. Precaution against respiratory complications is warranted in these patients, because they manifest with decreased CPF even in the presence of a positive cough.

\section{Methods}

This cross-sectional study was conducted at the Catholic University of Korea, Bucheon St. Mary's Hospital (Seoul, Korea), a university-affiliated hospital. Subjects who were recruited were first-ever subacute stroke patients ( 1 week to 1 month after stroke onset) who were treated at the stroke unit from September 1, 2013 to June 30,2014 . The local institutional review board of the hospital approved the study protocols (approval number HC13OISE0066). All participants or family members gave written consent.

\section{Subject Recruitment}

First-ever stroke patients were eligible for enrollment if they had unilateral hemiplegia caused by a single brain lesion, which was confirmed by brain computed tomography or magnetic resonance imaging. Subjects were recruited who lacked a cough response during aspiration (group A; penetration-aspiration score $=8$ ),${ }^{20}$ as confirmed through videofluoroscopic swallowing studies or fiberoptic endoscopic evaluation of swallowing. Subjects with no 


\section{Diaphragm Excursion During Reflexive Cough in Subjects With Stroke}

evidence of dysphagia who consumed a full normal oral diet with a functional oral intake scale ${ }^{21}$ score of 7 at the time of enrollment were the control group (group B). All participants in group B underwent instrumental evaluation of swallowing at the time of enrollment to confirm the absence of any silent aspiration (ie, penetration-aspiration score $=1) .{ }^{20}$

The exclusion criteria for enrollment were episodes of pulmonary embolism or dyspnea at the time of enrollment; cardiovascular comorbidities, such as myocardial infarction, congestive heart failure, uncontrolled arrhythmia, or hypertension; and a history of asthma exacerbations, frequent nausea, vomiting, or reflux episodes. Patients were also excluded if they had previous episodes of chest wall trauma that could affect diaphragmatic excursions or tracheostomy tubes, which can affect the degree of aspiration and penetration. ${ }^{22}$ Patients with severe dementia with a Mini-Mental State Examination (MMSE) score $<10$ were excluded.

\section{Clinical Variables}

Demographic features, such as brain lesion site and laterality, days since onset of stroke, and medical comorbidities of diabetes mellitus, hypertension, and smoking history, were recorded. In addition, a history of intubation within 1 week of admission to the acute stroke care unit was recorded. The initial National Institutes of Health Stroke Scale score upon admission to the stroke unit within $24 \mathrm{~h}$ of stroke was recorded. To assess the relationship between cough strength and other functional parameters reflecting the degree of hemiparesis, the following were obtained at the time of enrollment: modified Barthel index, trunk control test, MMSE, and Berg balance scale. ${ }^{23}$ Grip strength of the hemiplegic arm was assessed using the hand hydraulic Jamar hand dynamometer (Apsun, Songapu Ogumdong, Seoul, Korea), following standardized position and procedures, and the mean of the 3 trial values was used for analysis ${ }^{24}$ When hemiparesis was too profound and the grip strength values were unobtainable, the value 0 was recorded and used for analysis. These parameters were obtained by an occupational therapist who was blinded to the subjects' coughing ability. Enrolled subjects and their caregivers were monitored at our hospital during the initial 6 months after the onset of stroke. After enrollment, subjects were instructed to report for additional events related to respiratory complications after discharge. Medical electronic data were reviewed at the end of the study to record any events related to aspiration pneumonia that required admission to the pulmonology department or antibiotic treatment.

\section{Spirometry Measurements}

The FVC, $\mathrm{FEV}_{1}$, and $\mathrm{FEV}_{1} / \mathrm{FVC}$ were obtained from spirometry measurements (Vmax Autobox V62J) (Apsun).

Static maximum pressures during inspiration $\left(\mathrm{P}_{\text {Imax }}\right)$ and expiration $\left(\mathrm{P}_{\mathrm{Emax}}\right)$ were obtained using a respiratory pressure meter (Micro-Plus Spirometer, Carefusion, San Diego, California) with a standard flange mouthpiece. The highest value after 3 attempts was used for analysis. ${ }^{25}$ These measurements were performed by a physiatrist, based on guidelines proposed by the American Thoracic Society/European Respiratory Society.

\section{Assessment of Cough Strength}

Cough strength was measured in cough peak flow (CPF; L/min) using a peak flow meter (micropeak, Carefusion) under 2 conditions: during citric acid-induced reflexive cough and during voluntary cough when the subjects coughed at the examiner's command. Citric acid-induced reflexive cough was used as the primary outcome variable in this study. Voluntary cough was measured using the same procedures cited in a previous publication in accordance with the American Thoracic Society/European Respiratory Society statement. ${ }^{25}$ The values are presented as the mean of the 3 highest values of 5 attempts.

The citric acid-induced reflexive cough was evaluated, based on steps described in the literature..$^{3,12}$ A citric acid concentration of $0.28 \mathrm{~mol} / \mathrm{L}$ ( $5 \%$ citric acid) diluted in $0.9 \%$ sodium chloride was administered to all subjects for $20 \mathrm{~s}$ via a mouth mask. An ultrasonic nebulizer (NE-U17 nebulizer, Omron, Kyoto, Japan) was connected via an oral-nasal interface that was connected to a peak flow meter to record the cough strength, based on methods described previously. 5,26 Induced cough was recorded on at least 3 occasions simultaneously when the diaphragm excursion was assessed with sonography. Each reflexive cough trial was repeated after a resting interval $(30 \mathrm{~s}$ to $3 \mathrm{~min}$ ) to avoid habituation or tachyphylaxis. ${ }^{27}$ If no cough occurred in at least 2 of 3 assessments, the subject was recorded as lacking a cough response and considered a non-responder. ${ }^{12}$ For subjects showing successive cough responses after citric acid inhalation, the strength of the first cough, which has the strongest force, ${ }^{28}$ was used for analysis. The number of coughs generated after citric acid nebulization was counted. Following previous protocols, ${ }^{12}$ only coughs produced within $15 \mathrm{~s}$ of citric acid inhalation were recorded for analysis. A clinician blind to the subjects' demographic data evaluated the cough and respiratory function.

\section{Diaphragm Assessment}

Diaphragm excursion during citric acid-evoked reflexive cough and voluntary cough was recorded with a 


\section{Diaphragm Excursion During Reflexive Cough in Subjects With Stroke}

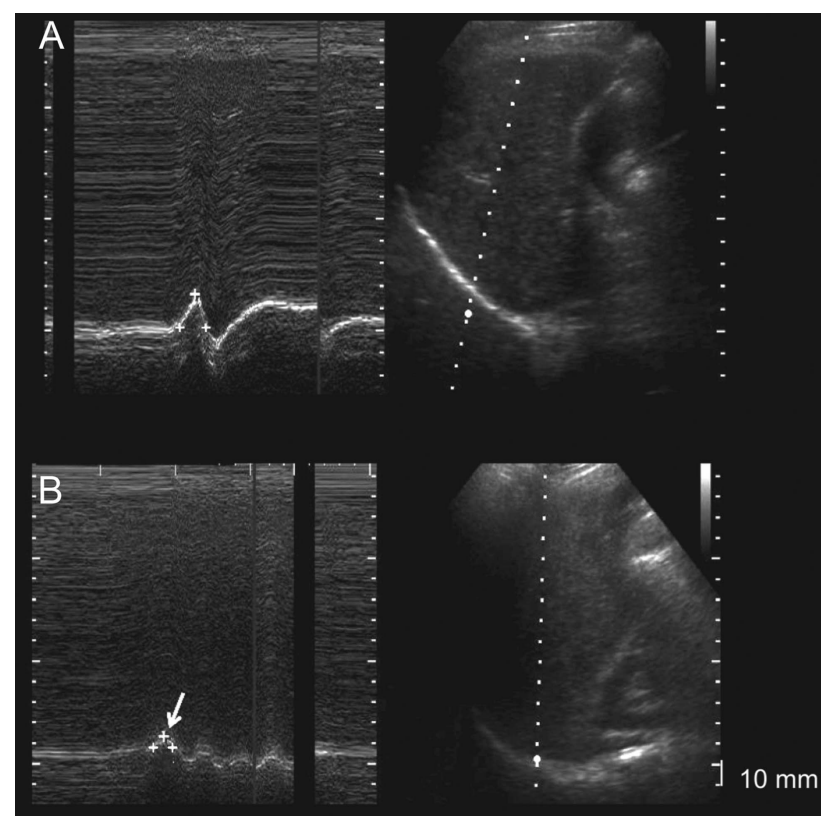

Fig. 1. Standard measurement method for diaphragm excursion tracing. A: The amplitudes of diaphragm excursion are measured using one caliper placed at the baseline of the diaphragm echoic line and a second caliper placed at the apex or the maximum height of the line in $\mathrm{cm}$. B: In subjects who generated multiple coughs during the citric acid reflexive cough test, the first diaphragm excursion (arrow) visualized is used to determine the degree of excursion amplitude. The degree of diaphragm excursion is measured from the baseline to the peak, as indicated by the cross symbols.

HD11XE ultrasound machine (Philips Healthcare, Andover, Massachusetts) in M-mode using a convex transducer $(5-2 \mathrm{MHz})$ with variable frequency (depending on the depth), based on methods described previously. ${ }^{7}$ In brief, the transducer was positioned just below the ribs between the midaxillary and the mammillary line with a $45^{\circ}$ angle tilt between the surface of the abdominal wall in the cephalic direction. All images were recorded in the longitudinal semicoronal plane through a subcostal or intercostal approach by $\mathrm{M}$-mode ultrasonography during a few respiratory cycles and different coughing maneuvers. All images were based on posterolateral regions, where the movements are known to show the greatest amplitudes. ${ }^{6}$ Both hemidiaphragms were evaluated, with the movement on each side recorded in centimeters. During reflexive cough, when more than one cough was generated, the degree of diaphragm excursion generated during the first cough was used for analysis (Fig. 1). The measurements were performed, based on previous research, ${ }^{7}$ with one caliper placed at the baseline of the diaphragm echoic line and a second caliper placed at the apex or the maximum height of the line. Sonography was performed by a physiatrist with $>5$ y of experience conducting diaphragm sonography imaging in stroke patients.

\section{Data Analysis}

Statistical analyses were performed using SAS 9.4 statistical software (SAS Institute, Cary, North Carolina). R 2.15.3 package software (R Foundation for Statistical Computing, Vienna, Austria) was used to produce the graphs. Values of $P<.05$ were significant.

Based on previous studies, ${ }^{26}$ sample size calculation revealed that a minimum of 21 participants in each group would be needed to detect a mean difference in the CPF during reflexive cough of $39 \mathrm{~L} / \mathrm{min}$ between subjects with dysphagia and a normal control group with a 5\% significance level and a power of $80 \%$. For subjects who showed absence of cough with citric acid induction, the values were defined as 0. Differences in clinical, functional, and spirometry findings between the 2 groups were analyzed using the chi-square test for categorical variables and the Wilcoxon rank-sum test for continuous variables.

The Wilcoxon rank-sum test was used to compare spirometric and sonography findings during voluntary cough and reflexive cough between the 2 groups. The correlation between the clinical variables and cough strength during both reflexive cough and voluntary cough was determined by the Spearman correlation coefficient. Additional subgroup analysis within group A was performed between subjects with and without prior intubation episodes in CPF and diaphragm excursions.

\section{Results}

\section{Participants}

Among 258 first-ever stroke patients admitted to the department of rehabilitation medicine who had previously received acute treatment at the stroke center of the authors' institution, 44 consecutively enrolled subjects fulfilled the inclusion criteria (Fig. 2). Two subjects dropped out before the evaluation process; one subject was excluded because of an unexpected early discharge, and one subject was excluded because of an acute skin reaction unrelated to the study. Therefore, a final total of 21 participants in each group completed the study. Demographic and clinical characteristics of these 2 groups showed no statistical differences, except for the trunk control test (Table 1). The study and control groups were not different in the proportion of individuals with diabetes mellitus (28.6\% vs $28.6 \%$ ), hypertension ( $71.4 \%$ vs $52.3 \%$ ), COPD ( $0 \%$ vs $0 \%)$, or smoking history ( $47.6 \%$ vs $66.6 \%)$. The median (interquartile range) functional oral intake scale level in group A was 2 (1-3) and was significantly different from the functional oral intake scale level in group B, in which all subjects had an functional oral intake scale score of 7 . In group A, 4 subjects had an intubation history within their first week of admission to the stroke unit. The 6-month 


\section{Diaphragm Excursion During Reflexive Cough in Subjects With Stroke}

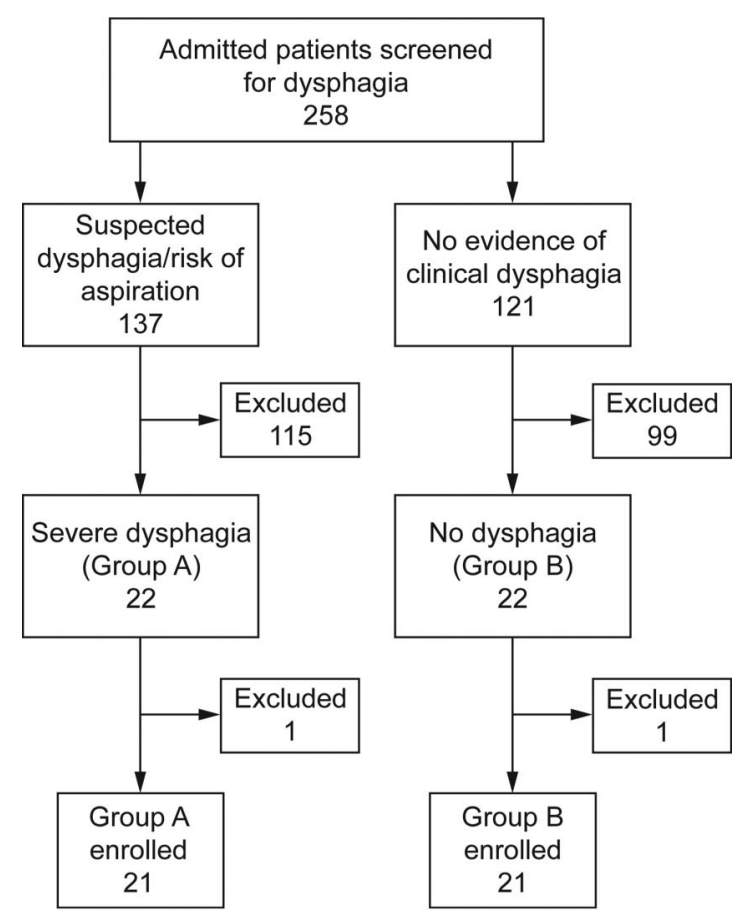

Fig. 2. Flow chart. follow-up revealed that 7 subjects in group A experienced aspiration pneumonia, which required antibiotic treatment.

\section{Spirometric and Cough Peak Flow Findings}

The 2 groups had significant differences in the $\mathrm{FEV}_{1}$, FVC, $\mathrm{P}_{\text {Imax }}$, and $\mathrm{P}_{\text {Emax }}$. Group A subjects also showed significantly reduced CPF (expressed as the median [interquartile range]), compared with group $\mathrm{B}$, during citric acid-induced reflexive cough (45.0 [0-83] L/min vs 97.0 [66-162] L/min, $P=.004$ ) (Fig. 3A) and during voluntary cough $(108$ [80-170] L/min vs 204.0 [153.0-236.0] L/min, $P<.001$ ) (Fig. 3B). Cough was not evoked in 8 subjects from group A (ie, non-responders), despite 3 citric acid challenge attempts. After excluding these non-responders, the CPF of citric acid-induced reflexive cough was significantly reduced in group A compared with group B (74 [48-97] L/min vs 97.0 [66-162.0] L/min, $P=.042$ ). Group A also had a smaller median (interquartile range) number of coughs (1 [0-2]) compared with group B (3 [2-5]) $(P<.001)$. A separate subgroup analysis of the nonresponders revealed that they had reduced $\mathrm{CPF}$ during voluntary cough compared with responders (Table 2).

Table 1. Differences in the Clinical Characteristics Between the 2 Stroke Groups

\begin{tabular}{|c|c|c|c|}
\hline Variable & Group A $(n=21)$ & Group B $(n=21)$ & $P$ \\
\hline \multicolumn{4}{|l|}{ Diagnosis-related } \\
\hline Stroke onset, mean $\pm \mathrm{SD} d$ & $14.67 \pm 6.21$ & $14.05 \pm 5.33$ & .83 \\
\hline Age, mean \pm SD y & $56.86 \pm 10.63$ & $59.57 \pm 10.22$ & .34 \\
\hline Sex (female), $n(\%)$ & $8(38.10)$ & $6(28.57)$ & .51 \\
\hline $\mathrm{BMI}$, mean $\pm \mathrm{SD} \mathrm{kg} / \mathrm{m}^{2}$ & $23.68 \pm 3.39$ & $23.97 \pm 2.94$ & .86 \\
\hline Right brain lesion, $n(\%)$ & $8(38.10)$ & $14(66.67)$ & .062 \\
\hline \multicolumn{4}{|l|}{ Etiology, $n(\%)$} \\
\hline Infarct & $12(57.14)$ & $14(66.67)$ & .75 \\
\hline Hemorrhage & $9(42.8)$ & $7(33.3)$ & .75 \\
\hline \multicolumn{4}{|l|}{ Location, $n(\%)$} \\
\hline Cortical & $4(19.05)$ & $3(14.29)$ & .52 \\
\hline Subcortical & $11(52.38)$ & $12(57.14)$ & $>.99$ \\
\hline Posterior circulation & $7(33.33)$ & $6(28.57)$ & .73 \\
\hline \multicolumn{4}{|c|}{ Functional background, mean $\pm \mathrm{SD}$} \\
\hline Initial NIHSS & $6.76 \pm 5.57$ & $4.48 \pm 4.56$ & .12 \\
\hline Total motricity index & $62.2 \pm 32.6$ & $61.3 \pm 26.2$ & .68 \\
\hline Trunk control test & $62.7 \pm 42.2$ & $87.8 \pm 25.5$ & .036 \\
\hline Berg & $28.0 \pm 26.5$ & $32.1 \pm 19.8$ & .48 \\
\hline MBI & $52.5 \pm 34.1$ & $61.9 \pm 27.7$ & .42 \\
\hline MMSE & $22.9 \pm 6.43$ & $25.4 \pm 5.06$ & .12 \\
\hline Hemiside grip strength & $11.5 \pm 10.56$ & $11.2 \pm 9.8$ & .95 \\
\hline \multicolumn{4}{|c|}{$\begin{array}{l}\text { Group A is the group of stroke subjects with confirmed dysphagia and silent aspiration and a penetration aspiration score of } 8 \text {. Group B is the group of stroke subjects without dysphagia or signs of } \\
\text { aspiration and a penetration-aspiration score of } 1 \text {. The } P \text { values for the differences between group A and group B were analyzed using the chi-square test and Wilcoxon rank-sum test with the } \\
\text { statistical significance set at } \alpha=.05 . \\
\text { BMI = body mass index } \\
\text { NIHSS = National Institutes of Health Stroke Scale } \\
\text { MBI = modified Barthel index } \\
\text { MMSE = Mini-Mental State Examination }\end{array}$} \\
\hline
\end{tabular}



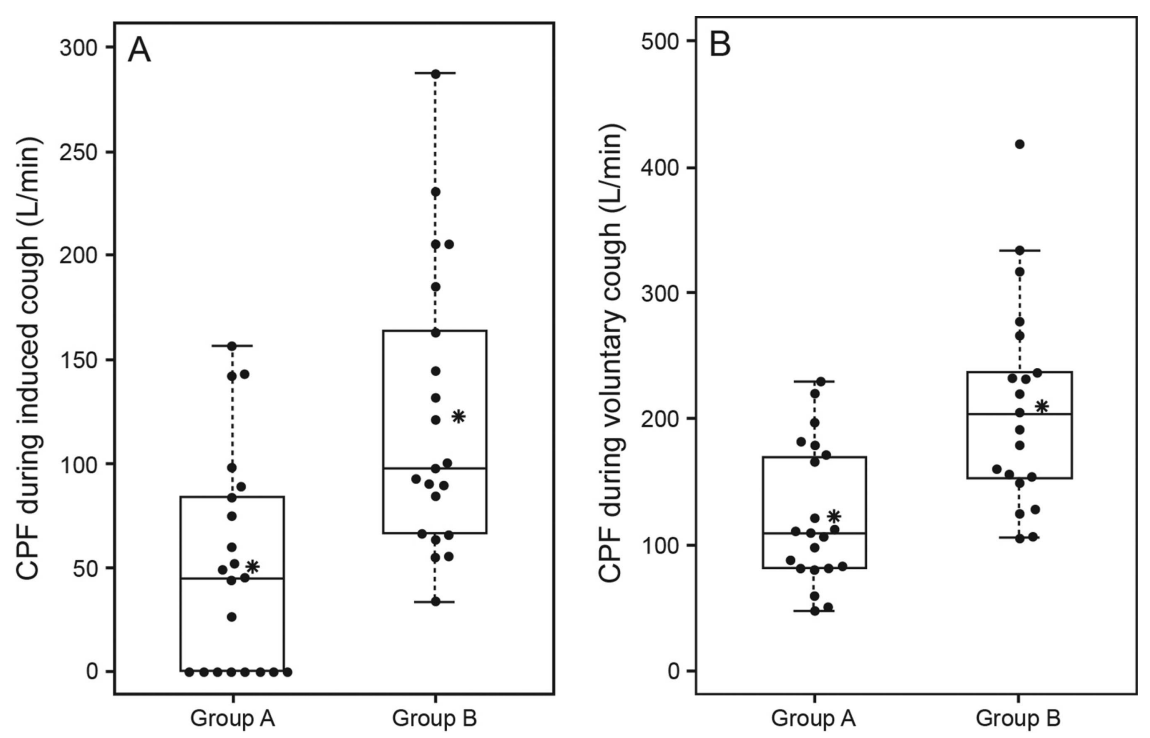

Fig. 3. Beeswarm boxplots of cough peak flows (CPF) in the 2 groups. A: The median (interquartile range) values of the CPF during citric acid-induced reflexive cough in group A $(n=21)$ are significantly smaller than those in group B $(n=21)(P=.004)$. B: The median (interquartile range) values of CPF during voluntary cough testing in group $\mathrm{A}$ are significantly smaller than those in group $\mathrm{B}(P<.001)$. The boxplots show the medians and quartiles. Single measurements are presented as black dots. Asterisks denote the mean value, and whiskers indicate the lowest to maximum measurements. Group A represents post-stroke subjects with dysphagia and silent aspiration after excluding non-responders in the citric acid cough test. Group B represents post-stroke subjects with no evidence of aspiration or dysphagia.

\section{Diaphragm Findings}

All subjects underwent diaphragm sonography without complications. Images were successfully obtained under different coughing maneuvers. The extents of diaphragm excursion during voluntary cough and reflexive cough detected by sonography were significantly different (Table 3), with group A subjects showing compromised diaphragm excursions on the hemiplegic and nonhemiplegic side during both cough types. During reflexive cough, the extent of diaphragm excursion was more markedly reduced in group A than in group B (Fig. 4). Subjects with prior intubation episodes showed no differences in diaphragm excursion during voluntary cough, compared with nonintubated subjects, although more severe stroke severity and reduced expiratory muscle forces were recorded (Table 2).

\section{Correlation Analysis}

The reflexive cough CPF showed a moderate positive correlation (Fig. 5) with the voluntary cough $\mathrm{CPF}\left(\mathrm{r}_{\mathrm{s}}=0.85\right.$, $P<.001)$, number of coughs $\left(\mathrm{r}_{\mathrm{s}}=0.69, P<.001\right)$, and degree of diaphragm excursion recorded for reflexive cough on the hemiplegic and nonhemiplegic sides $\left(\mathrm{r}_{\mathrm{s}}=0.50\right.$ and $\mathrm{r}_{\mathrm{s}}=0.55$, respectively; $P<.001$ ). The voluntary cough $\mathrm{CPF}$ had a weak-to-moderate positive correlation with the number of reflexive coughs produced $\left(\mathrm{r}_{\mathrm{s}}=0.54, P<.001\right)$ and the degree of diaphragm excursion during voluntary cough on the hemiplegic and nonhemiplegic sides $\left(\mathrm{r}_{\mathrm{s}}=0.50\right.$ and $\mathrm{r}_{\mathrm{s}}=0.39$, respectively; $P<.001$ ). In addition, the voluntary cough CPF showed positive correlation with the parameters that reflected the degree of a patient's cooperation, alertness, and volitional control of the truncal and expiratory muscles, the $\mathrm{P}_{\text {Emax }}\left(\mathrm{r}_{\mathrm{s}}=0.50, P<.001\right)$, truncal control index $\left(\mathrm{r}_{\mathrm{s}}=0.45\right.$, $P=.003)$, and MMSE results $\left(\mathrm{r}_{\mathrm{s}}=0.32, P=.040\right)$. However, neither the CPF values obtained during reflexive cough $\left(\mathrm{r}_{\mathrm{s}}=-0.16, P=.32\right)$ or voluntary cough $\left(\mathrm{r}_{\mathrm{s}}=-0.189\right.$, $P=.23)$ were significantly correlated with the National Institutes of Health Stroke Scale score.

\section{Discussion}

This study showed that stroke subjects at risk of aspiration had significantly reduced $\mathrm{CPF}$ of $45 \mathrm{~L} / \mathrm{min}$ with impaired diaphragm excursion during the citric acid cough test. Compared with the control group of stroke subjects with no risk of aspiration, reduced cough strength occurred, despite similarities in stroke severity, degree of hemiparesis, and functional deficits between the 2 groups. The CPF produced during the citric acid reflexive cough test was positively correlated with the degree of diaphragm excursion and the CPF generated during voluntary cough. To the best of our knowledge, this was the first study to demonstrate impaired diaphragm excursion during citric acid-induced reflexive cough with reduced CPF values in stroke subjects at risk of aspiration. With impaired dia- 


\section{Diaphragm Excursion During Reflexive Cough in Subjects With Stroke}

Table 2. Differences in the Basic Clinical Characteristics, Respiratory Pressure Meter, and Sonography Findings Between the Responders and Non-Responders to the Citric Acid Reflexive Cough Test and Between Subjects With Prior Intubation and No Intubation History in Group A

\begin{tabular}{|c|c|c|c|c|}
\hline \multirow{2}{*}{ Variables } & \multicolumn{2}{|c|}{ Response to the Citric Acid-Induced Cough Test } & \multicolumn{2}{|c|}{ Prior Intubation History } \\
\hline & Non-Responder $(n=8)$ & Responder $(n=13)$ & Intubation $(n=4)$ & No intubation $(n=17)$ \\
\hline Age, $y$ & $58.5(53.0-64.5)$ & $56.0(47.0-62.7)$ & $52(47-59)$ & $58(52-65.5)$ \\
\hline Initial NIHSS & $3.5(3-8.5)$ & $6(3-10.0)$ & $16.5(12.5-19.5)$ & $3(3-6)^{*}$ \\
\hline MBI & $69.0(28.5-90.0)$ & $50.0(12.5-76.5)$ & $10(2.5-56.5)$ & $59(31.7-85.0)$ \\
\hline MMSE & $25.5(22.1-28.8)$ & $21.3(16.9-25.6)$ & $14(7-25.0)$ & $25(21.5-28.0)$ \\
\hline $\mathrm{P}_{\text {Imax }}, \mathrm{cm} \mathrm{H}_{2} \mathrm{O}$ & $37(12.0-69.0)$ & $24(17.5-53.0)$ & $12.5(2.5-35.0)$ & $36.0(17.5-56.0)$ \\
\hline $\mathrm{P}_{\text {Emax }}, \mathrm{cm} \mathrm{H}_{2} \mathrm{O}$ & $59.5(29.5-69.0)$ & $30(13.2-60.0)$ & $10.5(6.5-33.0)$ & $55.0(29.2-64.7)^{*}$ \\
\hline $\mathrm{CPF} \mathrm{VC}, \mathrm{L} / \mathrm{min}$ & $79.5(54.5-92.0)$ & $165.0(108.7-184.5) \dagger$ & $108.0(80-166.2)$ & $131.5(64.5-200)$ \\
\hline $\mathrm{CPF} \mathrm{RC}, \mathrm{L} / \mathrm{min}$ & NA & NA & $37(13-68)$ & $45(0-86.5)$ \\
\hline Hemiside diaphragm $\mathrm{VC} \mathrm{cm}$ & $1.7(1.5-2.4)$ & $1.7(1.4-2.1)$ & $1.7(1.4-2.1)$ & $1.7(1.4-2.2)$ \\
\hline Nonhemi side diaphragm $\mathrm{VC} \mathrm{cm}$ & $2.5(2.0-3.0)$ & $3.1(1.8-3.7)$ & $1(0.5-1.5)$ & $1(0-2)$ \\
\hline $\begin{array}{l}\text { Results are median (interquartile range). } \\
* P<.05 \text {. The } P \text { value is based on the Mann } \\
\dagger P<.05 \text {. The } P \text { value is based on the Mant } \\
\text { NIHSS = National Institutes of Health Stroke } \\
\text { MBI = modified Barthel Index } \\
\text { MMSE = Mini-Mental State Examination } \\
\mathrm{P}_{\text {Imax }}=\text { static maximum pressure during insp } \\
\mathrm{P}_{\text {Emax }}=\text { static maximum pressure during exp } \\
\text { CPF = cough peak flow } \\
\text { NA = not applicable } \\
\text { VC = voluntary cough } \\
\text { RC = reflexive cough induced by citric acid }\end{array}$ & $\begin{array}{l}\text { ey } U \text { test with the statistical signif } \\
\text { ey } U \text { test with the statistical signif }\end{array}$ & $\begin{array}{l}\text { t at } \alpha=.05 . \text { The comparison is } \\
\text { tat } \alpha=0.05 \text {. The comparison }\end{array}$ & $\begin{array}{l}\text { ntubation versus nonintuba } \\
\text { onders versus non-responde }\end{array}$ & \\
\hline
\end{tabular}

Table 3. Differences in Spirometric and Sonography Findings of the Diaphragm During Voluntary and Reflexive Coughing Between the 2 Groups

\begin{tabular}{|c|c|c|c|c|c|}
\hline \multirow{2}{*}{ Variable } & \multicolumn{2}{|c|}{ Group A $(n=21)$} & \multicolumn{2}{|c|}{ Group B $(n=21)$} & \multirow{2}{*}{$P$} \\
\hline & Mean \pm SD & Median (IQR) & Mean \pm SD & Median (IQR) & \\
\hline \multicolumn{6}{|l|}{ Spirometry and respiratory measurement } \\
\hline $\mathrm{FEV}_{1}, \%$ predicted & $63.7 \pm 17.8$ & $63(51.0-71.0)$ & $79.8 \pm 14.4$ & $81(69.7-86.0)$ & .008 \\
\hline FVC, $\%$ predicted & $72.0 \pm 19.4$ & $75(61.0-80.0)$ & $91.1 \pm 13.1$ & $86(82.7-102)$ & .003 \\
\hline $\mathrm{FEV}_{1} / \mathrm{FVC}(\%)$ & $82 \pm 8$ & $80(74-89)$ & $84 \pm 10$ & $81(75-91)$ & .62 \\
\hline $\mathrm{P}_{\text {Imax }}, \mathrm{cm} \mathrm{H}_{2} \mathrm{O}$ & $36.71 \pm 28.31$ & $35.0(16.0-53.0)$ & $56.19 \pm 28.76$ & $55.0(46.0-62.0)$ & .043 \\
\hline $\mathrm{P}_{\text {Emax }}, \mathrm{cm} \mathrm{H}_{2} \mathrm{O}$ & $43.76 \pm 25.98$ & $43.00(23.00-64.00)$ & $65.05 \pm 28.15$ & $62.0(48.0-71.0)$ & .02 \\
\hline \multicolumn{6}{|l|}{ Diaphragm sonography, cm } \\
\hline \multicolumn{6}{|l|}{ Reflexive cough } \\
\hline Hemiplegic side & $1.0 \pm 0.8$ & $1.4(0.0-1.6)$ & $2.1 \pm 0.6$ & $2.0(1.8-2.4)$ & $<.001$ \\
\hline Nonhemiplegic side & $1.3 \pm 1.09$ & $1.6(0.0-1.9)$ & $2.5 \pm 0.7$ & $2.5(2.1-2.9)$ & $<.002$ \\
\hline \multicolumn{6}{|l|}{ Voluntary cough } \\
\hline Hemiplegic side & $1.9 \pm 0.6$ & $1.7(15-2.2)$ & $2.7 \pm 0.7$ & $2.7(2.4-2.9)$ & $<.001$ \\
\hline Nonhemiplegic side & $2.7 \pm 0.8$ & $2.6(1.9-3.3)$ & $3.7 \pm 1.0$ & $3.6(2.9-4.6)$ & .003 \\
\hline $\begin{array}{l}\text { The } P \text { value is based on the Wilcoxon rank-sum test } \\
\mathrm{IQR}=\text { interquartile range } \\
\mathrm{P}_{\text {Emax }}=\text { static maximum pressure during expiration } \\
\mathrm{P}_{\text {Imax }}=\text { static maximum pressure during inspiration }\end{array}$ & tatistical significance & $x=.05$ & & & \\
\hline
\end{tabular}

phragm recruitment during reflexive cough and severely weakened coughs, these subjects may ultimately experience more respiratory complications.

Our study results are in accordance with previous studies $^{4,11-13,26,29}$ that have advocated the use of the re- flexive cough test to detect individuals at risk of silent aspiration. In contrast to past studies that define the strength response of a cough test with subjective parameters, ${ }^{27}$ such as strong, weak, or absent, which are only somewhat reliable, ${ }^{28}$ our study attempted to record 


\section{Diaphragm Excursion During Reflexive Cough in Subjects With Stroke}

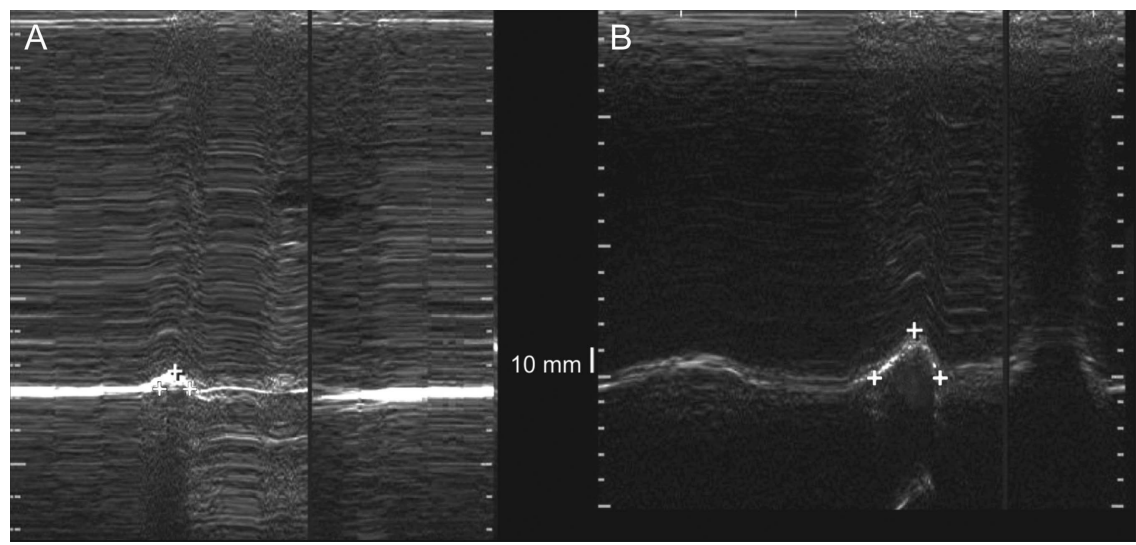

Fig. 4. Diaphragm excursions in waveforms obtained from M-mode tracings during citric acid-induced reflexive cough and recorded from the hemiplegic sides. A: Very limited diaphragm excursion $(0.76 \mathrm{~cm})$ in a subject with post-stroke dysphagia and silent aspiration. B: A post-stroke subject with no dysphagia or aspiration. The degree of diaphragm excursion $(2.09 \mathrm{~cm})$ is greater than that of the subject in A. The degree of diaphragm excursion is measured from the baseline to the peak, as indicated by the cross symbols.

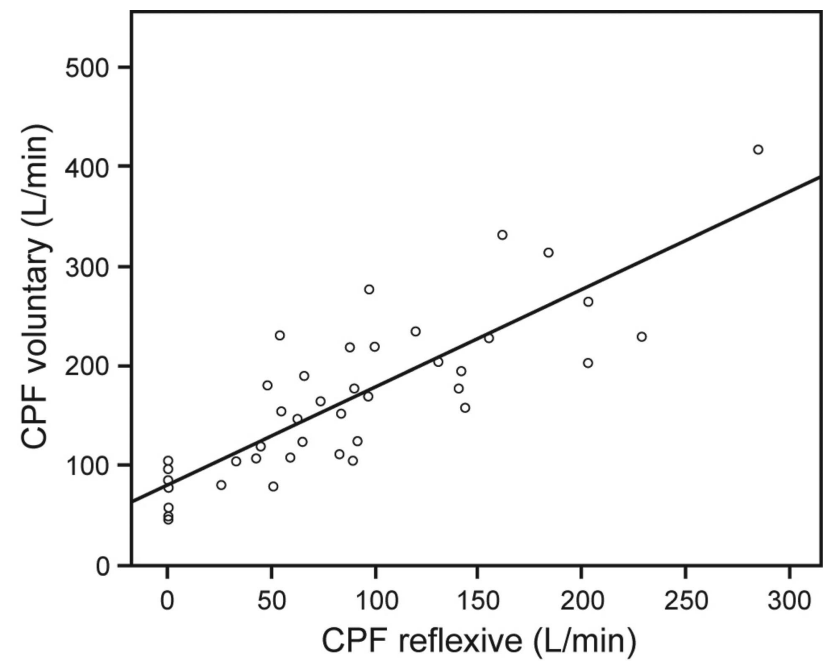

Fig. 5. Scatter plot indicating significant correlation between voluntary cough strength and reflexive cough strength, as measured by the cough peak flow (CPF).

these responses in CPF. Similar attempts to record CPF have been made in stroke subjects, ${ }^{5,26}$ although the previous subjects were either heterogenic in nature ${ }^{23}$ or did not have swallowing disturbances assessed by instrumental means ${ }^{5}$; therefore, direct comparison of the cough strength in subjects with confirmed silent aspiration versus those with only stroke, as in our study, could not be made.

Cough sensitivity and cough strength are 2 different neurophysiologic processes, ${ }^{28}$ but impairment in either process increases the risk of pneumonia. Our study suggests the need to measure cough strength objectively with CPF, especially in patients with increased risk of aspiration during swallowing. The citric acid test is usually performed at different concentrations to measure decreased cough sen- sitivity in stroke patients ${ }^{6,26}$; however, there are wide interindividual variances in this cough sensitivity. In addition, cough sensitivity may vary, based on the onset of stroke: cough thresholds tested at the first week after stroke onset differ from those at the fourth week post-stroke. ${ }^{30}$ Cough strength via CPF at fixed dosages may help to obtain objective measurements of protective cough with less variance in stroke subjects.

Reflexive cough is related to a cough during swallowing; therefore, information on this CPF parameter could be clinically relevant. As shown in the subgroup analysis of group A after excluding the non-responders, even subjects who coughed with citric acid and would be positive responders by conventional standards, the CPF levels generated were still comparatively lower than in group B subjects. Cough sensitivity and decreased CPF generated reflexively during aspiration may predispose these patients to increased risk of respiratory complications. Various studies have already reported on the diagnostic properties of the reflexive cough test based on cough sensitivity, but the diagnostic properties of the CPF during reflexive cough still need to be established. For example, during voluntary cough, a CPF cut-off value $<160 \mathrm{~L} / \mathrm{min}$ is used to identify individuals with an ineffective cough. ${ }^{29}$ Similar cutoff values in reflexive cough would allow screening of patients who may be at risk of weak cough strength during swallowing and be at increased risk of respiratory complications.

Weakness of the inspiratory muscles may prevent the lungs from reaching high volumes, which may decrease cough effectiveness by putting the expiratory muscles at a mechanical disadvantage. The diaphragm, which is a major inspiratory muscle, can be affected after acute stroke. Past studies with M-mode sonography have shown that stroke subjects are known to exhibit pronounced weakness 


\section{Diaphragm Excursion During Reflexive Cough in Subjects With Stroke}

of the diaphragm as assessed by M-mode sonography, with a more restrictive lung dysfunction. ${ }^{7}$ This involvement can be bilateral, affecting even the nonhemiparetic side. ${ }^{15,16}$ Whereas past studies ${ }^{7}$ have already shown that stroke subjects show compromised diaphragm excursions during voluntary cough after a stroke, this study is clinically relevant for showing markedly reduced cough strength with reduced recruitment of the diaphragm muscles during the reflexive cough test in individuals at risk of silent aspiration.

The diaphragm, although it is the primary inspiratory muscle, is important in cough because it generates a strong positive intrathoracic pressure and greater expiratory flow during its compressive phase. To date, limited studies exist on how the diaphragm is affected during reflexive cough after stroke. To the best of our knowledge, this is one of the first studies to demonstrate impaired recruitment and demonstrate that CPF during reflexive cough is correlated with the degree of diaphragm excursion in subjects at risk of silent aspiration. Expiratory muscle recruitment during coughing was not directly assessed; however, reduced maximum expiratory pressure and differences in the trunk control test scores occurred. The latter finding implies impaired abdominal or paraspinalis muscle control related to cough and may indicate the impairment in the expiratory muscles.

In addition, the $\mathrm{CPF}$ during reflexive cough showed strong correlation with the number of coughs and with voluntary cough. Unlike in previous reports, ${ }^{4}$ the reflexive cough showed no correlation with the degree of weakness or functional disability, as measured by the National Institutes of Health Stroke Scale score, Barthel index, or grip strength.

These findings differed from those of the voluntary cough, which showed positive correlations with the truncal index, $\mathrm{P}_{\text {Emax }}$, and MMSE values. Whereas voluntary cough is mediated by the cortex and dependent on volitional efforts and cognitive function, reflexive cough is primarily mediated by the brainstem, ${ }^{4}$ which may explain these discrepancies. The reflexive cough is not affected by conscious effort, although its strength can be used to reflect the voluntary cough strength. Therefore, estimation of the CPF during reflexive cough may be used as a surrogate marker of cough ability in severely affected stroke patients who may be unable to cooperate to produce a voluntary cough on command. ${ }^{26}$

Our results support findings from previous studies ${ }^{10,18,19}$ that demonstrate an intricate relationship between decreased pharyngeal sensory afferents and respiratory muscle activation during reflexive cough. Previous studies ${ }^{2}$ of laryngectomized subjects, who lack afferent information from the larynx, have shown that they manifest impaired mechanisms subserving the motor unit recruitment rate and motor output of the expiratory muscles during reflexive cough. The topic of how swallowing and respiration are interconnected or whether these processes are disparate is still under debate. However, according to our study, the integrity of the pharyngeal afferents may be the key factor to link these 2 functions.

The feasibility of providing pharyngeal afferents to lead to better cough function has been suggested recently, ${ }^{31}$ where successful decannulation was shown in stroke subjects who underwent pharyngeal electrical stimulation, a novel therapy that stimulates pharyngeal sensory afferents, and showed improved secretion management. Therefore, in consideration of the close interconnections between swallowing and protective reflexive cough, it may be tentatively postulated that providing sensory input to the impaired pharyngeal afferents may also stimulate the neural control of cough as well as the swallowing performance. Whether this novel pharyngeal stimulation therapy leads to improved cough strength and better respiratory muscle recruitment during reflexive cough, including the diaphragm, needs to be further explored. In consideration of the equivocal and mixed results reported in clinical trials ${ }^{31}$ for respiratory training, such as expiratory muscle strengthening and voluntary cough training in stroke patients, this novel technique of providing pharyngeal stimulation could be a useful alternative therapy to improve cough functions in patients at risk of aspiration.

Some limitations in this study require consideration. The concentration of citric acid used in this study was different from those employed in previous publications. ${ }^{11,13}$ The aim of this study was not to measure cough sensitivity, but to evoke coughing in subjects at risk of silent aspiration. To ensure that a cough could be elicited in all subjects, a higher dose than that of the initial proposed protocol was required. ${ }^{13}$ Even at the higher dose, some subjects failed to demonstrate an reflexive cough. The discrepancies in concentration across different studies ${ }^{4,11,13,26,32}$ reflect the heterogenic methods that are employed in current citric acid cough testing. Another discrepant finding from past studies ${ }^{2,5}$ was that more severe subjects with high National Institutes of Health Stroke Scale values may have been excluded because only subjects without a history of tracheostomy who were fully cooperative in following the evaluations were enrolled. The median MMSE values of group A and B were 22.9 and 25.4, which indicated that subjects were fully cooperative and cognitively alert to perform the respiratory pressure meter evaluations and cough assessments. Future studies with more severe neurologically involved subjects are needed in addition to studies that directly assess expiratory muscle recruitment using surface electromyography. Another point is that our study involved a small number of subjects with a prior intubation history. This group showed more severe neurological deficits and higher National Institutes of Health Stroke Scale scores; however, this subgroup analysis needs to be interpreted with caution. The effect of prior intubation on the CPF during 


\section{Diaphragm Excursion During Reflexive Cough in Subjects With Stroke}

reflexive cough is a topic that needs assessment in future studies. A final point is that the cough center is at the brainstem level, ${ }^{33}$ and therefore patients with infratentorial lesions affecting this center would show more compromised cough function. ${ }^{14}$ However, our results were unable to demonstrate whether CPF was more limited in individuals with infratentorial lesions than in individuals with supratentorial lesions. The effect of different brain lesions on reflexive cough is a topic that needs to be addressed in future studies.

Nevertheless, the results have important clinical implications for patients with a high risk of silent aspiration. Our study was performed in a homogeneous group of subjects with subacute stroke and confirmation of silent aspiration through instrumental assessments. The results of our study may be applicable to stroke patients with different chronicity and other swallowing disorders that may involve impaired respiratory muscle recruitment. Our results emphasize the need to assess the CPF of reflexive cough in patients at high risk of aspiration.

\section{Conclusions}

In summary, our results revealed that, in a subgroup of subacute stroke subjects with risk of aspiration, CPF was significantly reduced, and diaphragm excursion was impaired during a reflexive cough test, even in the presence of a positive cough sign. During swallowing training, a clinician may need to consider weakened cough strength and reduced diaphragm excursion during reflexive cough and voluntary cough in individuals at risk of silent aspiration, despite showing positive cough signs during the reflexive cough test.

\section{REFERENCES}

1. Hinchey JA, Shephard T, Furie K, Smith D, Wang D, Tonn S. Formal dysphagia screening protocols prevent pneumonia. Stroke 2005;36(9):1972-1976.

2. Lasserson D, Mills K, Arunachalam R, Polkey M, Moxham J, Kalra L. Differences in motor activation of voluntary and reflex cough in humans. Thorax 2006;61(8):699-705.

3. Addington WR, Stephens RE, Gilliland KA. Assessing the laryngeal cough reflex and the risk of developing pneumonia after stroke: an interhospital comparison. Stroke 1999;30(6):1203-1207.

4. Widdicombe JG, Addington WR, Fontana GA, Stephens RE. Voluntary and reflex cough and the expiration reflex; implications for aspiration after stroke. Pulm Pharmacol Ther 2011;24(3):312-317.

5. Kulnik ST, Birring SS, Hodsoll J, Moxham J, Rafferty GF, Kalra L. Higher cough flow is associated with lower risk of pneumonia in acute stroke. Thorax 2016;71(5):474-475.

6. Smith Hammond CA, Goldstein LB, Zajac DJ, Gray L, Davenport $\mathrm{PW}$, Bolser DC. Assessment of aspiration risk in stroke patients with quantification of voluntary cough. Neurology 2001;56(4):502-506.

7. Park GY, Kim SR, Kim YW, Jo KW, Lee EJ, Kim YM, Im S. Decreased diaphragm excursion in stroke patients with dysphagia as assessed by M-mode sonography. Arch Phys Med Rehabil 2015; 96(1):114-121.
8. Hammond CAS, Goldstein LB. Cough and aspiration of food and liquids due to oral-pharyngeal dysphagia: ACCP evidence-based clinical practice guidelines. Chest 2006;129(1 Suppl):154S-168S.

9. Pitts T. Airway protective mechanisms. Lung 2014;192(1):27-31.

10. Fontana GA, Pantaleo T, Lavorini F, Mutolo D, Polli G, Pistolesi M. Coughing in laryngectomized patients. Am J Respir Crit Care Med 1999;160(5 Pt 1):1578-1584.

11. Guillén-Solà A, Chiarella SC, Martínez-Orfila J, Duarte E, AlvaradoPanesso M, Figueres-Cugat A, et al. Usefulness of citric cough test for screening of silent aspiration in subacute stroke patients: a prospective study. Arch Phys Med Rehabil 2015;96(7):1277-1283.

12. Miles A, Moore S, McFarlane M, Lee F, Allen J, Huckabee ML. Comparison of cough reflex test against instrumental assessment of aspiration. Physiol Behav 2013;118:25-31.

13. Sato M, Tohara H, Iida T, Wada S, Inoue M, Ueda K. Simplified cough test for screening silent aspiration. Arch Phys Med Rehabil 2012;93(11):1982-1986.

14. Vilardell N, Rofes L, Nascimento WV, Muriana D, Palomeras E, Clave P. Cough reflex attenuation and swallowing dysfunction in sub-acute post-stroke patients: prevalence, risk factors, and clinical outcome. Neurogastroenterol Motil 2017;29(1). doi: 10.1111/nmo. 12910.

15. de Almeida IC, Clementino AC, Rocha EH, Brandão DC, Dornelas de Andrade A. Effects of hemiplegy on pulmonary function and diaphragmatic dome displacement. Respir Physiol Neurobiol 2011; 178(2):196-201.

16. Voyvoda N, Yucel C, Karatas G, Oguzulgen I, Oktar S. An evaluation of diaphragmatic movements in hemiplegic patients. Br J Radiol 2012;85(1012):411-414.

17. Pikus L, Levine MS, Yang YX, Rubesin SE, Katzka DA, Laufer I, Gefter WB. Videofluoroscopic studies of swallowing dysfunction and the relative risk of pneumonia. AJR 2003;180(6):1613-1616.

18. Bongianni F, Corda M, Fontana G, Pantaleo T. Influences of superior laryngeal afferent stimulation on expiratory activity in cats. J Appl Physiol 1988;65(1):385-392.

19. Jodkowski JS, Berger AJ. Influences from laryngeal afferents on expiratory bulbospinal neurons and motoneurons. J Appl Physiol 1988;64(4):1337-1345.

20. Rosenbek JC, Robbins JA, Roecker EB, Coyle JL, Wood JL. A penetration-aspiration scale. Dysphagia 1996;11(2):93-98

21. Crary MA, Mann GD, Groher ME. Initial psychometric assessment of a functional oral intake scale for dysphagia in stroke patients. Arch Phys Med Rehabil 2005;86(8):1516-1520.

22. Ledl C, Ullrich YY. Occlusion of tracheostomy tubes does not alter pharyngeal phase kinematics but reduces penetration by enhancing pharyngeal clearance: a prospective study in patients with neurogenic dysphagia. Am J Phys Med Rehabil 2017;96(4):268-272.

23. Berg K, Wood-Dauphinee S, Williams JI. The Balance Scale: reliability assessment with elderly residents and patients with an acute stroke. Scand J Rehabil Med 1995;27(1):27-36.

24. Dodds RM, Syddall HE, Cooper R, Kuh D, Cooper C, Sayer AA. Global variation in grip strength: a systematic review and metaanalysis of normative data. Age Ageing 2016;45(2):209-216.

25. American Thoracic Society/European Respiratory Society. ATS/ERS statement on respiratory muscle testing. Am J Respir Crit Care Med 2002;166(4):518-624.

26. Lee SC, Kang SW, Kim MT, Kim YK, Chang WH, Im SH. Correlation between voluntary cough and laryngeal cough reflex flows in patients with traumatic brain injury. Arch Phys Med Rehabil 2013; 94(8):1580-1583.

27. Morice AH, Fontana GA, Belvisi MG, Birring SS, Chung KF, Dicpinigaitis PV, et al. ERS guidelines on the assessment of cough. Eur Respir J 2007;29(6):1256-1276. 


\section{Diaphragm Excursion During Reflexive Cough in Subjects With Stroke}

28. Britton D, Benditt JO, Merati AL, Miller RM, Stepp CE, Boitano L, et al. Associations between laryngeal and cough dysfunction in motor neuron disease with bulbar involvement. Dysphagia 2014;29(6):637-646.

29. Ward K, Seymour J, Steier J, Jolley CJ, Polkey MI, Kalra L, Moxham J. Acute ischaemic hemispheric stroke is associated with impairment of reflex in addition to voluntary cough. Eur Respir J 2010;36(6):1383-1390.

30. Kobayashi H, Hoshino M, Okayama K, Sekizawa K, Sasaki H. Swallowing and cough reflexes after onset of stroke. Chest 1994;105(5): 1623.
31. Hegland KW, Davenport PW, Brandimore AE, Singletary FF, Troche MS. Rehabilitation of swallowing and cough functions following stroke: an expiratory muscle strength training trial. Arch Phys Med Rehabil 2016;97(8):1345-1351.

32. Miles A, Huckabee ML. Intra- and inter-rater reliability for judgement of cough following citric acid inhalation. Int J Speech Lang Pathol 2013;15(2):209-215.

33. Bolser DC, Pitts TE, Davenport PW, Morris KF. Role of the dorsal medulla in the neurogenesis of airway protection. Pulm Pharmacol Ther 2015;35:105-110. 